\title{
Surface instability of Kelvin-Helmholtz type in Couple stress fluid layer bounded above by a porous layer and below by rigid surface
}

\author{
${ }^{1}$ Krishna B. Chavaraddi, ${ }^{2}$ V.B. Awati and ${ }^{3}$ Priya M. Gouder \\ ${ }^{1}$ Department of Mathematics, Government First Grade College, Yellapur (U.K)-581 359, Karnataka, India \\ ${ }^{2}$ Department of Mathematics, Rani Channamma University, Belgaum-590020, Karnataka, India \\ ${ }^{3}$ Department of Mathematics, K.L.E's Dr. M. S. Sheshagiri College of Engineering and Technology, \\ Belgaum-590008, Karnataka, India
}

\begin{abstract}
The surface instability of Kelvin-Helmholtz type in a couple stress fluid layers bounded above by a porous layer and below by a rigid surface is investigated using linear stability analysis. A simple theory based on fully developed flow approximations is used to derive the dispersion relation for the growth rate of KHI in presence of couple stress fluid. In order to observe the effect of boundary layer applying the Beavers-Joseph (BJ) slip condition. The dispersion relation is derived using suitable boundary and surface conditions and the results are discussed through graphically. The couple stress fluid is found to be stabilizing effect and the influence of the various parameters of the problem on the interface stability is thoroughly analyzed.
\end{abstract}

Key Words: Couple-stress fluid, KHI, B-J Condition, dispersion relation, porous media.

\section{Introduction}

Kelvin-Helmholtz instability after Lord Kelvin(1910) and Hermann Von Helmholtz (1868) can occur when velocity shear is present within a continuous fluid or when there is sufficient velocity difference between two fluids. One example is wind blowing over a water surface where the wind causes the relative motion between the stratified layers (i.e., water and air). Helmholtz(1868) studied the dynamics of two fluids of different densities when a small disturbance such as a wave is introduced at the boundary connecting the fluids. If surface tension can be ignored and for some short enough wavelengths, two fluids in parallel motion with different velocities and densities yielded an interface that is unstable for all speeds. The KHI is important in understanding a variety of space and astrophysical, geophysical phenomena involving plasma flow. Without surface tension, this streaming is unstable no matter how small the velocity difference between the layers may be. It was shown by Kelvin[1910] that the surface tension will suppress the instability if the difference in velocity is sufficient small. From an industrial view point, the momentum transfer and the KHI in composite region provides impetus for effective, design of porous bearings in lubrication process, particularly in the slider bearings and in the effective design of target in inertial fusion energy(IFE). Because of these importances the $\mathrm{KHI}$ is investigated in this paper using the linear stability analysis. The experimental observation of KHI has been given by Francis(1954). The linear study of the KHI was investigated by Chandrasekhar(1961) gave an introduction to classical KHI. He discussed the effect of surface tension, variable density, orotation and applied magnetic field on the behaviour of stability.

The study of electrohydrodynamic(EHD) KHI of free surface charges, separating two semi-infinite dielectric fluids and influence by an electric field has been discussed by Melcher(1963). The effect of rotation and a general oblique magnetic field on the KHI has been studied by Sharma and Srivastava(1968). The main difference between KH and RT instabilities is inclusion of $(\vec{q} \cdot \nabla) \vec{q}$ which is a nonlinear term in the perturbation equations. EHD studies the interplay of mechanical and electrical forces in fluids. In the first approximation it is assumed that the electrical currents are very weak and therefore magnetic effects are negligible. Maxwell's equations are then reduced to Gauss' law and the charge conservation law. Within the past few years, a number of paper have appeared on studies of surface instability in the presence of electric field (Moatimid and El-Dib(1996)). Singh and Khare(1991) have studied the stability of two semi-infinite homogenous gravitating streams of infinite conductivity under uniform horizontal magnetic field and uniform rotation. Bhatia and Hazarika(1995) were discussed this stability for superposed viscous gravitating fluids. The importance of the KHI problem has been demonstrated by Benjamin and Bridges (1997) who have given an excellent reappraisal of the classical KHI problem in hydrodynamics. They have shown that the problem admits of canonical Hamiltonian formulation and obtained several new results. 
The KHI of two superposed viscous fluids in a uniform vertical magnetic field is discussed in the presence of effects of surface tension and permeability of porous medium by Bhatia and Sharma (2003). Following Babchin et al(1984) and Rudraiah et al (1996), a simple theory based on Stokes and lubrication approximations is used in this study by replacing the effect of the boundary layer with a Beavers and Joseph(1967) slip condition with the primary objective of using porous layer to suppress the growth rate of KHI. In the above studied the fluid has been considered to be Newtonian. Nevertheless, much attention has not been given in the literature to the study of KHI in a poorly conducting non-Newtonian fluid like Couple stress fluid layer bounded above by a porous material and below by rigid surface that in spite of frequently occurring in many engineering and physical situations namely, inertial fusion energy (IFE), geophysics and supernova, the consideration of such fluids is desirable. The couple-stress effects are considered as result of the action of one part of a deforming body on its neighbourhood. Stokes (1966) has formulated the theory of a couple-stress fluid. The theory of Stokes (1966) allows for the polar effects such as the presence of couple-stresses and body couples and has been applied to the study of some simple lubrication problems (see Sinha et al.(1981), Bujurke and Jayaraman(1982)). According to Stokes (1966), couple-stresses appear in fluids with very high molecules. Since the long chain hylauronic acid molecules are found as additives in synovial fluids, Walicki and Walicka(1999) were modeled synovial fluid as couple-stress fluid in human joints. The presence of small amounts of additives in a lubricant can improve the bearing performance by increasing the lubricant viscosity and thus producing an increase in the load capacity. This additive in a lubricant also reduces the coefficient of friction and increases the temperature range in which bearing can operate. The RTI of two superposed infinitely conducting couple-stress fluids of uniform densities in a porous medium in the presence of a uniform magnetic field by Sunil et al.,(2002). It is clear notice that the couple-stress fluid in region -1 with proper choice of couple-stress parameter and porous parameter in reducing the asymmetry of the two fluid composite systems at the interface. The electrorheological KHI of fluid sheet have been discussed by El-Dib and Matoog (2005). Due to the rather complicated nature of the problem a mathematical simplification is considered where the weak effects of viscoeleastic fluids are taken into account. Recently, Chavaraddi et al (2011) have investigated the effect of KHI of fluid layer bounded above by a porous layer and below by a rigid surface in presence of magnetic field. It is clear that magnetic field is found to be stabilizing effect and the influence of the various fluid parameters of the problem on the interface stability.

Keeping in mind the importance of non-Newtonian (couple-stress) fluids in modern technology and industries as well as various applications mentioned above, the objective of the predict to study the KHI in a poorly conducting couple-stress fluid layer bounded above by a porous layer and below by a rigid surface in presence of effects of surface tension using BJ condition at the interface and the plan of this paper is as follows. The mathematical formulation subjected to the boundary and surface conditions is given in Section 2. The expression for the dispersion relation is derived using the basic equations with boundary and surface conditions in section 3. The cutoff and maximum wave numbers and the corresponding maximum growth rate are also obtained in section 4 and some important conclusions are drawn in final section of this paper.

\section{Mathematical Formulation}

The physical configuration is shown in Fig.1. The system under consideration is composed of two incompressible couple-stress fluid and fluid saturated porous media separated by the plane $y=h$. The above fluid is of infinite extent while the below one is of finite extent with rigid boundary. We take $y=h$ at the mean level of the interface and the y-axis pointing vertically upwards into the upper fluid. The fluids in both the regions are set in relative motion by acceleration normal to the interface and small perturbations are amplified when fluid in the thin film to the heavy fluid saturated porous media above the interface. The interface between couple stress fluid and fluid saturated porous media is described by $\eta(x, t)$ as the perturbed interface between two fluids in region-1 and 2. The two fluids are influenced by a couple-stress and porous parameters which acting along the positive $\mathrm{x}$-direction, where the $u_{f}$ and $u_{p}$ along the positive $\mathrm{x}$-direction, where the $\mathrm{x}$-axis is mean level of the wave. Also, the fluids are streaming with velocities $u_{f}$ and $u_{p}$ long the same direction. Acceleration due to gravity acts in the negative y-direction. A schematic diagram of the configuration in the steady state is given in Figure-1. 


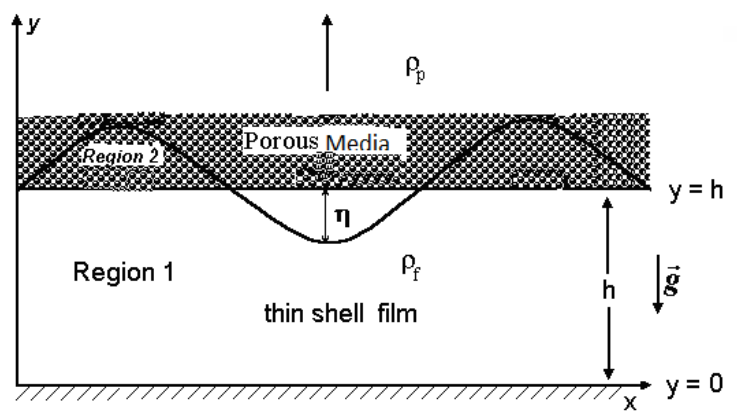

Fig. 1: Schematic diagram of the system

The upper fluid has density $\rho_{p}$ while the lower one has density $\rho_{f}$ and surface tension exist between the two fluids and is denoted by $\gamma$.

To investigate the problems posed in the paper the following combined lubrication and Stokes approximations are used.

(i) The clear dense liquid is homogeneous and isotropic.

(ii) The film thickness $h$ is much smaller than the thickness $H$ of the porous layer bounded above the film. That is,

$$
h<<H .
$$

(iii) The Strouhal number $S$ is assumed to be negligibly small.

(iv) The surface elevation $\eta$ is assumed to be small compared to film thickness $h$. That is,

$$
\eta<<h \text {. }
$$

(v) Nonuniform polarization and electric charge injection are negligible.

(vi) The fluid viscosity and thermal conductivity are assumed to be constants.

Following these assumptions and approximations, the basic equations are

\section{Region-1 :}

$$
\begin{aligned}
& \nabla \cdot \vec{q}=0 \\
& \rho\left(\frac{\partial \vec{q}}{\partial t}+(\vec{q} \cdot \nabla) \vec{q}\right)=-\nabla p+\mu \nabla^{2} \vec{q}-\lambda \nabla^{4} \vec{q}
\end{aligned}
$$

Region-2 :

$$
Q=-\frac{k}{\mu} \frac{\partial p}{\partial x}
$$

where $\vec{q}=(u, v)$ the fluid velocity, $\lambda$ the couple-stress parameter, $\varepsilon_{e}$ the dielectric constant, $p$ the pressure, $\frac{\partial p}{\partial x}$ the pressure gradient, $\mu$ the fluid viscosity, $Q=(Q, 0,0)$ the uniform Darcy velocity and $\rho$ the fluid density.

Let us non-dimensionalize the equations using

$x^{*}=\frac{x}{h}, y^{*}=\frac{y}{h}, u^{*}=\frac{u}{\delta h^{2} / \mu}, v^{*}=\frac{v}{\delta h^{2} / \mu}, p^{*}=\frac{p}{\delta h}, Q^{*}=\frac{Q}{\delta h^{2} / \mu}, t^{*}=\frac{t}{\delta h / \mu}$

Following the assumptions and approximations as stated above (i.e., Stokes and lubrication approximations), assuming that the heavy fluid in the porous layer is almost static because of creeping flow approximation and substituting Eq.(2.4) into Eqs.(2.1) and (2.2), we obtain (after neglecting the asterisks for simplicity)

Region-1:

$$
0=\frac{\partial u}{\partial x}+\frac{\partial v}{\partial y}
$$




$$
\begin{aligned}
& 0=-\frac{\partial p}{\partial x}+\frac{\partial^{2} u}{\partial y^{2}}-M_{0}^{2} \frac{\partial^{4} u}{\partial y^{4}} \\
& 0=-\frac{\partial p}{\partial y}
\end{aligned}
$$

Region-2 :

$$
Q=-\frac{1}{\sigma_{p}^{2}} \frac{\partial p}{\partial x}
$$

where $M_{0}=\sqrt{\lambda / \mu h^{2}}$ is the couple-stress parameter and $\sigma_{p}=\frac{h}{\sqrt{k}}$ the porous parameter.

\section{Dispersion Relation}

To find the dispersion relation, first we have to find the velocity distribution from Eq. (2.6) using the following boundary and surface conditions in addition to couple-stress boundary conditions.

(i) no-slip condition

$$
u=0 \quad \text { at } \quad y=0
$$

(ii) B-J condition

$$
\frac{\partial u}{\partial y}=-\alpha_{p} \sigma_{p}\left(u_{B}-Q\right) \text { at } \quad y=1
$$

where $\quad u=u_{B} \quad$ at $\quad y=1$

(iii) Couple-stress conditions:

$$
\frac{\partial^{2} u}{\partial y^{2}}=0 \quad \text { at } \quad y=0 \& 1
$$

(iv) Kinematic condition: $v=\frac{\partial \eta}{\partial t}$ at $y=1$

(v) Dynamic condition: $\quad p=-\eta-\frac{1}{B} \frac{\partial^{2} \eta}{\partial x^{2}}$ at $y=1$.

where $B=\delta h^{2} / \gamma$ is the Bond number and $\eta=\eta(x, y, t)$ is the elevation of the interface.

The solution of (2.6) subject to the above conditions is

$$
u=C_{1}+C_{2} y+C_{3} \cosh \left(y / M_{0}\right)+C_{4} \operatorname{Sinh}\left(y / M_{0}\right)+\frac{P}{2 M_{0}} y^{2}
$$

where

$$
\begin{aligned}
& P=\frac{\partial p}{\partial x}, \quad C_{1}=P M_{0}, \\
& C_{2}=\frac{-C_{1}}{\left(1+\alpha_{p} \sigma_{p}\right)}\left[\alpha_{p} \sigma_{p}-\left\{\frac{1}{M_{0}} \operatorname{Sinh}\left(1 / M_{0}\right)+\alpha_{p} \sigma_{p} \operatorname{Cosh}\left(1 / M_{0}\right)\right\}\right. \\
& \left.C_{3}=-C_{4}\left\{\frac{1}{M_{0}} \operatorname{Cosh}\left(1 / M_{0}\right)+\alpha_{p} \sigma_{p} \operatorname{Sinh}\left(1 / M_{0}\right)\right\}+\left\{\frac{1}{M_{0}^{2}}+\frac{1}{2 M_{0}^{3}} \alpha_{p} \sigma_{p}+\frac{\alpha_{p}}{\sigma_{p} M_{0}}\right\}\right] \\
& C_{4}=C_{1}\left[\operatorname{Coth}\left(1 / M_{0}\right)-\operatorname{Cosech}\left(1 / M_{0}\right)\right]
\end{aligned}
$$

After integrating Eq.(2.5) with respect to $y$ between $y=0$ and 1 and using Eq.(3.6), we get 


$$
v(1)=v_{1}=-\int_{0}^{1} \frac{\partial u}{\partial x} d y=\frac{\partial^{2} p}{\partial x^{2}} N
$$

where

$$
N=C_{1}+\frac{C_{2}}{2}+C_{3} M_{0} \operatorname{Sinh}\left(1 / M_{0}\right)+C_{4} M_{0}\left\{\operatorname{Cosh}\left(1 / M_{0}\right)-1\right\}+\frac{1}{6 M_{0}}
$$

Then Eq.(3.4), using Eqs.(3.5) and (3.7), becomes

$$
\frac{\partial \eta}{\partial t}=-\left[\frac{\partial^{2} \eta}{\partial x^{2}}+\frac{1}{B} \frac{\partial^{4} \eta}{\partial x^{4}}\right] N
$$

To investigate the growth rate, $n$, of the periodic perturbation of the interface, we look for the solution of Eq.(3.8) in the form

$$
\eta=\eta(y) \exp \{i \ell x+n t\}
$$

where $\ell$ is the wave number and $\eta(y)$ is the amplitude of perturbation of the interface.

Substituting Eq.(3.9) into Eq.(3.8), we obtain the dispersion relation in the form

$$
n=\ell^{2}\left(1-\frac{\ell^{2}}{B}\right) N .
$$

In the absence of couple-stress parameters, that is $M_{0} \rightarrow 0$, the growth rate given

by Eq.(3.10) reduces to $n_{b}$. Now the dispersion formula can be expressed in the form

$$
n=n_{b}-\ell \beta v_{a}
$$

where

$$
n_{b}=\frac{\ell^{2}}{3}\left(1-\frac{\ell^{2}}{B}\right), v_{a}=N \ell\left\{1-\frac{\ell^{2}}{B}\right\}, \quad \beta=\frac{\left(\frac{1-3 N}{3 N}\right)\left(1-\frac{\ell^{2}}{B}\right)}{\left(1--\frac{\ell^{2}}{B}\right)} .
$$

Setting $n=0$ in Eq.(3.10), we obtain the cut-off wavenumber, $\ell_{c t}$ in the form

$$
\ell_{c t}=\sqrt{B}
$$

The maximum wavenumber, $\ell_{m}$ obtained from Eq.(3.10) by setting $\partial n / \partial \ell=0$ is

$$
\ell_{m}=\sqrt{\frac{B}{2}}=\frac{\ell_{c t}}{\sqrt{2}}
$$

The corresponding maximum growth rate, $n_{m}$ for applied voltage opposing gravity is

$$
n_{m}=\frac{B}{4} N
$$

Similarly, using $\ell_{m}=\sqrt{B / 2}$, we obtain

$$
n_{b m}=\frac{B}{12} \text {. }
$$


Therefore,

$$
G_{m}=\frac{n_{m}}{n_{b m}}=3 N
$$

The growth rate given by Equation (3.10) is computed numerically for different values of parameters and the results are presented graphically in Figures 2-4.

\section{Results and Discussion}

In this study we have shown the surface instability of KH type in a Couple stress fluid layers bounded above by a porous layer and below by a rigid surface. Numerical calculations were performed to determine the growth rate at different wavenumbers for various fluid properties like couple stress parameter $M_{0}$, Bond number $B$ and porous parameter $\sigma_{p}$. We have plotted the dimensionless growth rate of the perturbation against the dimensionless wavenumber for some of the cases only.

When we fix all the input parameters except the ratio of the Hartmann number $M$, we find that the higher the couple-stress parameter the more stable the interface is. In Figure 2, we have plotted the growth rate against the wavenumber in the case where $\alpha_{p}=0.1, \sigma_{p}=4, B=0.02$ for different values of the couplestress parameter $M_{0}$. Increasing the couple-stress ratio results in slightly increasing the critical wavenumber and decreasing the maximum growth rate this is because of the action of the body couples on the system. Thus it has a stabilizing effect for the selected values of input parameters due to the increased in the couple-stress parameter.

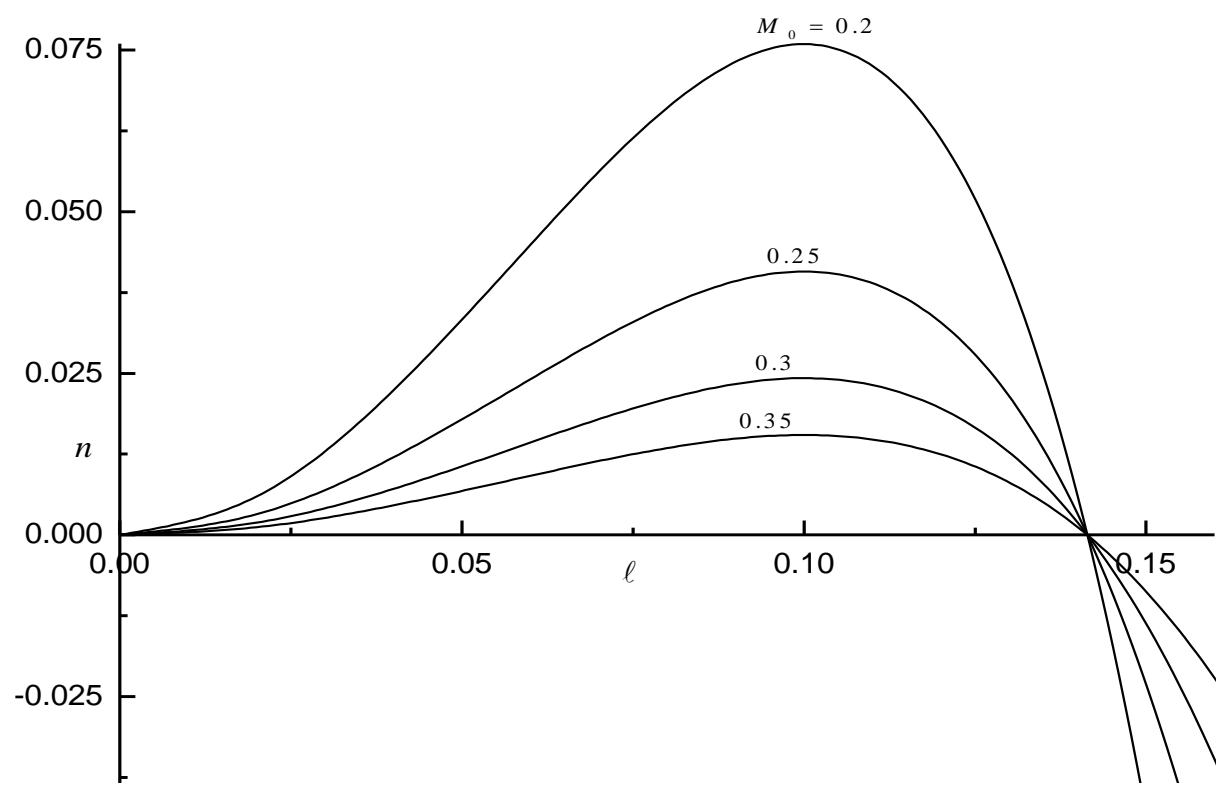

Figure 2: Growth rate, $n$ versus the wavenumber, $\ell$ for different values of couple stress parameter, $M_{0}$ when $\alpha_{p}=0.1, \sigma_{p}=4, B=0.02$. 


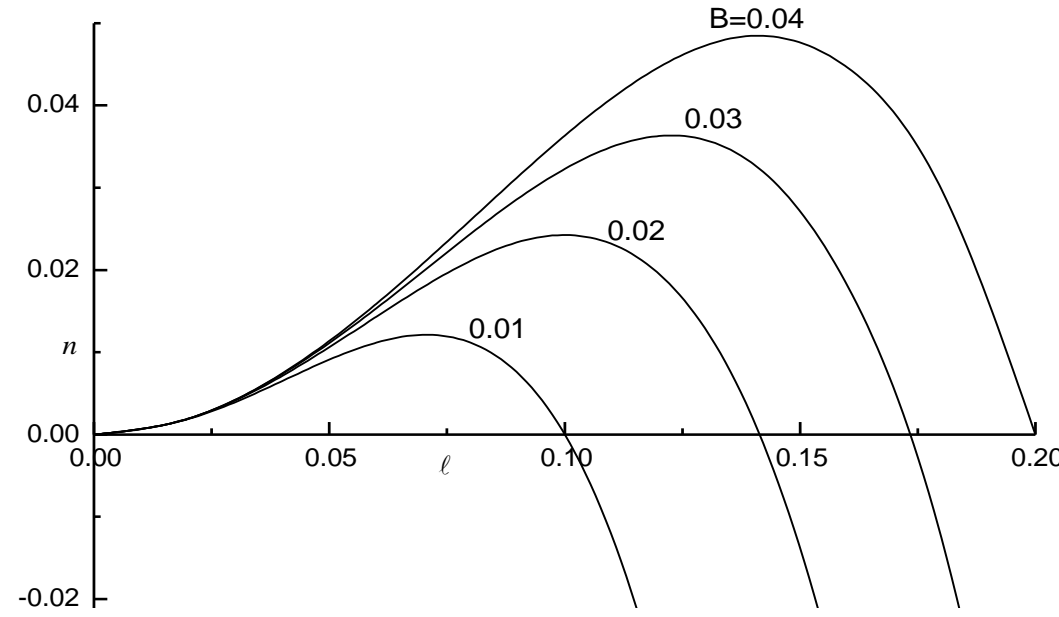

Figure 3: Growth rate, $n$ versus the wavenumber, $\ell$ for different values of Bond number $B$ when $\alpha_{p}=0.1, \sigma_{p}=4, M_{0}=0.3$.

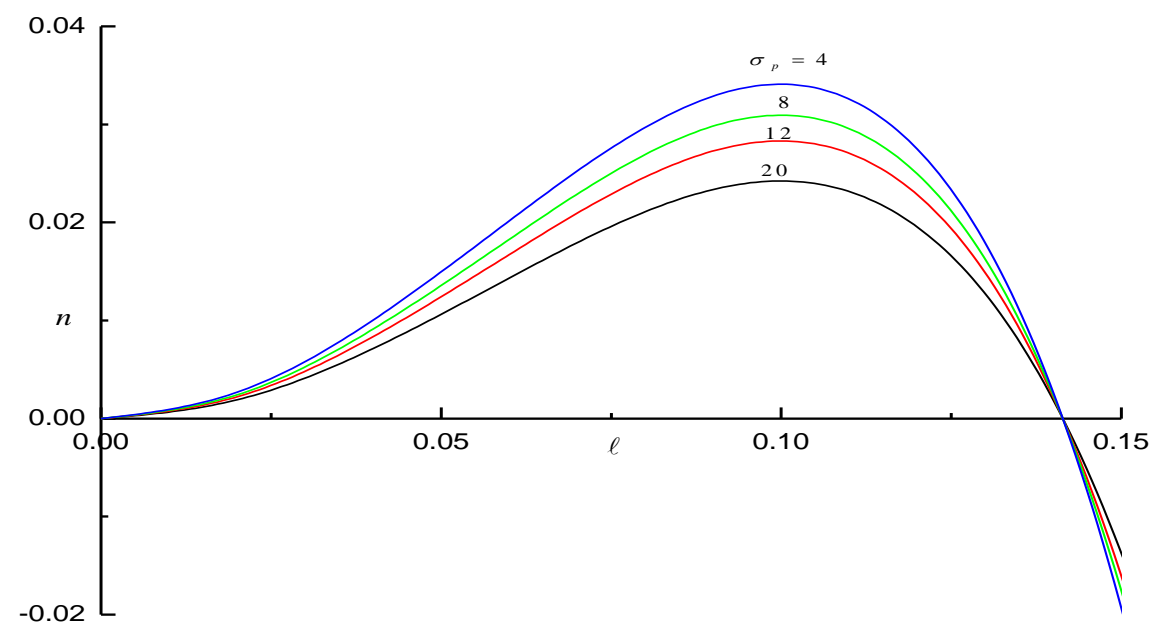

Figure 4: Growth rate, $n$ versus the wavenumber, $\ell$ for different values of porous parameter, $\sigma_{p}$ when $\alpha_{p}=0.1, M_{0}=0.3, B=0.02$.

In addition, we have investigated the effect of the surface tension of the fluid on the instability of the interface. In our sample calculations, we have taken $\alpha_{p}=0.1, \sigma_{p}=4, M_{0}=0.3$ and varied the Bond number $B$. For this input parameters, the critical wavenumber and maximum growth rate decreased as the ratio of the Bond number $B$ decreased from 0.4 to 0.1 as observed in Figure 3. The Bond number is reciprocal of surface tension and thus showing that an increase in surface tension decreases the growth rate and hence make the interface more stable.

However, in order to understand the effect of the porous properties on the instability, we now fix values of other parameters $\alpha_{p}=0.1, B=0.02$ and $M_{0}=0.3$ and vary the ratios of the porous parameters. Figure 4 displays the results of our calculations, showing that increasing the ratio of porous parameters $\sigma_{p}$ from 4 to 20 (and thus increasing the Darcy resistance compared to the viscous force) increases the critical wavelength and decreases the maximum growth rate, thus having a stabilizing effect by this parameter. We conclude that an increase in $\sigma_{p}$ also stabilizes the KHI due to the resistance offered by the solid particles of the porous layer to the fluid. 


\section{Acknowledgement}

The author (KBC) wishes to thank the Commissioner, Dept. of Collegiate Education, Government of Karnataka for their encouragement and support and one of the authors (VBA) acknowledge the U.G.C. (University Grant Commission, India) and thank for providing financial support for the research work. Also, the author (PMG) wishes to thank the Principal, KLE's Dr. M. S. Sheshagiri College of Engineering and Technology, Belgaum for their encouragement and support in doing research.

\section{References}

[1]. L. Kelvin(1910), Hydrokinetic solutions and observations , On the motion of free solids through a liquid, 69-75, “ Influence of wind and capillary on waves in water superposed frictionless, 76-85, Mathematical and Physical Papers IV, Hydrodynamics and General Dynamics, Cambridge, England.

[2]. H. Helmholtz (1868), Under discontinuirliche Flussigkeitsbewegungen, Wissenschaftliche Abhandlungen, 146-157 or Phil. Mag. Ser. 4(36), 337.

[3]. S. Chandrasekhar(1961), Hydrodynamic and Hydromagnetic Stability, Dover Publications, New York

[4]. J. R. D. Francis (1954), Wave motions and the aerodynamic drag on a free oil surface, Phil. Mag. Ser. 7, 45, 695.

[5]. Melcher, J. R., (1963), Field coupled surface waves, Cambridge, Massachussets, MIT Press.

[6]. Sharma, R.C., and Srivastava, K. M., (1968), Effect of horizontal and vertical magnetic fields on Rayleigh-Taylor instability, Aust. J. Phys., 21, 923.

[7]. G.M. Motamid, Y.O. El-Dib, Int. J. Theor. Phys. 35, 425 (1996).

[8]. R. P. Singh and H. C. Khare(1991), Stability of rotating gravitating superposed streams in a uniform magnetic field, Proc. Nat. Acad. Sci., City, vol.43,55.

[9]. P.K. Bhatia and A. B. Hazarika(1995), Gravitational instability of partially ionized plasma in an oblique magnetic field, Physica Scripts, Vol. 51(6), 775.

[10]. T. R. Benjamin and T. J. Bridges(1997), Reappraisal of the K-H problem, Part-2: Introduction of the Kelvin-Helmholtz superharmonic and Benjamin-Feir instabilities, J. Fluid Mech., 333, 327.

[11]. P. K. Bhatia and A. Sharma(2003), KHI of two viscous superposed conducting fluids, Proc. Nat. Acad. Sci. India, 73(A), IV

[12]. A. J. Babchin, A. L. Frenkel, B. G. Levich, G. I. Shivashinsky(1983), Nonlinear Saturation of Rayleigh-Taylor Instability in Thin Films, Phys. Fluids, 26, 3159.

[13]. N. Rudraiah, B. S. Krishnamurthy and R. D. Mathad(1996), The effect of oblique magnetic field on the surface instability of a finite conducting fluid layer, Acta, Mech., 119, 165.

[14]. G.S. Beavers and D.D. Joseph(1967), Boundary conditions at a naturally permeable wall, J. Fluid Mech., $30,197$.

[15]. V.K. Stokes(1966), Couple stresses in Fluids, Phys. Fluids. 9, 1709.

[16]. P. Sinha, P., C. Singh and K. R. Prasad(1981), Couple Stresses in Journal Bearings Lubricants and the Effect of Cavitations, WEAR, 67(1), 15

[17]. N. M. Bujurke, and G. Jayaraman(1982), The influence of couple stresses in squeeze films, Int. J. Mech. Sci., $24,369$.

[18]. E. Walicki and A. Walicka(1999), Flows of generalized second grade fluids in rectilinear channels, Appl. Mech. Engg. 4, 363.

[19]. Sunil, R.C., Sharma, and R. S. Chandel(2002), On superposed fluids in porous medium in hydrodynamics, Z. Naturforsch, 57a, 955.

[20]. Y. O. El-Dib and R. T. Matoog(2005), Electrorheological Kelvin-Helmholtz instability of a fluid sheet, Journal of Colloid and Interface Science 289, 223.

[21]. K. B. Chavaraddi, N. N. Katagi and V. B. Awati(2012), Kelvin-Helmholtz instability in a fluid layer bounded above by a porous layer and below by a rigid surface in presence of magnetic field, Applied Mathematics, 3, 564 . 